\title{
At-home Cosmeceutical Application and Outpatient Treatments: A 3D Stepwise Facial Rejuvenation Approach
}

\author{
Fabrizio Melfa ${ }^{1}$, Daniela Siragusa ${ }^{1}$, Daniela Caruso ${ }^{2}$, Carmelo Lo Faro ${ }^{3 *}$, Giovanni Francesco Nicoletti ${ }^{4}$, Raffaele Rauso ${ }^{5}$ \\ ${ }^{1}$ Department of Aesthetic Medicine, Private Practice, Palermo, Milano, Italy; ${ }^{2}$ Department of Aesthetic Medicine, Private Practice, \\ Catania, Milano, Italy; ${ }^{3}$ Department of Maxillofacial Surgery, University Federico II, Naples, Italy; ${ }^{4}$ Head of Plastic Surgery Unit, \\ University of Campania "Luigi Vanvitelli", Naples, Italy; ${ }^{5}$ Department of Maxillofacial Surgery, University of Campania "Luigi \\ Vanvitelli", Naples, Italy
}

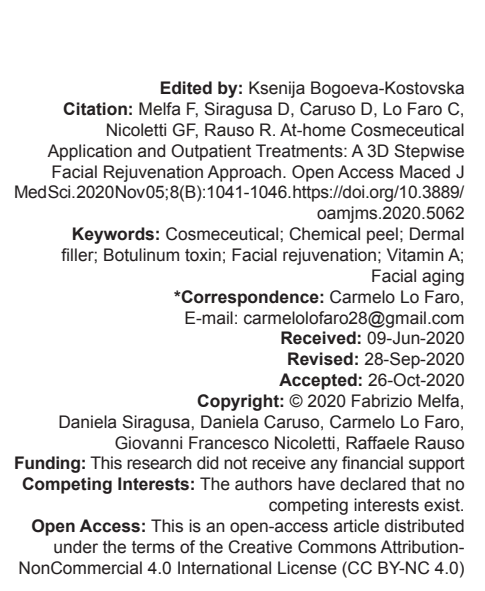

Introduction

Facial aging is the result of numerous genetic, actinic, and environmental factors. Over time, skin undergoes atrophic variations that produce folds and wrinkles. In addition, bone mass and fat volume tend to decrease, muscles become more flaccid, ligaments lose tension, and slipping of the dermal-epidermal layer occurs [1], [2]. Aging affects the 3-dimensional (3D) structure of all the facial tissues: bones, muscles, ligaments, adipose tissue, and skin [1], [2]. However, skin-related effects represent the most noticeable sign of aging for the patient [3].

The demand for non-invasive facial esthetic procedures has increased dramatically. In 2018, consumers spent more than 1 billion dollars on injectable treatments [4]. Injection of hyaluronic acid (HA) was the second most popular nonsurgical cosmetic procedure (after injection of botulinum toxin) with over 800,000 treatments performed, an almost 12\% increase from 2017 to 2018 and 60\% increase from 2014 to 2018 [4]. The increase in popularity of nonsurgical procedures could be explained, at least in part, by the affordability of these treatments and the minimal downtime associated with recovery.

In the present article, the authors present a holistic approach for facial rejuvenation in which multiple nonsurgical esthetic treatments are performed in a stepwise fashion to manage the aging face in a 3D manner; treatment plan is customized for each patient, although 2 main flow charts are followed based on patient facial aging degree.

This novel stepwise approach for facial rejuvenation entails treatment of the skin with cosmeceuticals (i.e., cosmetic products with medicinal or drug-like benefits) applied at home, followed by more involved cosmetic treatments (filler, toxins, threads, etc.) performed in an outpatient setting; this "double" approach empowers the patient having several follow-up appointments and different procedures can be performed, obtaining at the same time a mild approach for the patient, natural results, and patients retention. The key point of this stepwise facial approach is represented by treating facial structures superficial to deep: The skin is the first target. The at-home treatments address the dermal-epidermal skin layer 
and yield improvements in skin texture and dermal tonicity. Subsequent outpatient procedures address the layers of the face from deepest to most superficial. This technique has been termed "3D stepwise facial rejuvenation."

\section{Materials and Methods}

\section{Participants and study object}

This prospective study was performed in accordance with guidelines set forth in the Declaration of Helsinki. Forty-four patients (42 women, 2 men) provided informed consent and were evaluated in this study. Eligible patients were those who wished to undergo facial rejuvenation without surgery, by means of home and outpatient medical treatments and who followed the therapeutic program prescribed at the first medical consultation. Patients who did not wish to receive the therapies planned at the initial visit were excluded from the study, as were patients who required other pharmacologic medical treatments involving the face.

\section{Assessment of Skin Analysis}

Participating patients received medical assessments every 15-21 days and underwent diagnostic testing with an A-One Smart automated skin analysis system (Bomtech Electronics, Seoul, South Korea). The A-One Smart system enables photographic analysis of the skin by numeric algorithms applied to detect a change in specific variables such as skin elasticity and hydration, facial pores, and superficial wrinkles. The system also stores photographs obtained at each assessment. For each participant, the author analyzed skin hydration and elasticity, the number and density of pores, blemishes, superficial wrinkle, and the density and distribution of skin hyperpigmentation.

\section{Treatment}

All the patients received a three step treatment; in all the cases, as the first step, skincare treatments were prescribed to be performed daily at home, the second step was represented by chemical peel application, and as the third step, others procedures of esthetic medicine such as filler, botulinum toxin, threads, or energy-based devices (EBD) were tailored per each patient (Table 1).

The first step in 3D stepwise facial rejuvenation is represented by the application of cosmeceuticals by the patient at home. We empirically divided the patients in two groups: The first one with a low degree of facial aging (patients with superficial lines and/or skin spots and/or low degree of facial soft tissue ptosis, etc.); the second one with moderate/high degree of facial aging (deep facial folds and/or skin spots and/or medium-high degree of facial ptotic tissue, etc.). An example of a low or medium/high degree of facial aging is reported in Figures 1 and 2 .

For the first group daily, retinol $1 \%$ cream application at home, for a period from 6 to 8 weeks, was prescribed; after this period, we performed, as the second step, a 10\% TCA-based peeling (3 Step Stimulation Peel by ZO Medical; Health Inc. - Irvine, $\mathrm{CA})$. After that, at least 1 month later, we started the third step, evaluating the need for injectables, such as botulinum toxin type A-BoNTA- (incobotulinum toxin $50 \mathrm{UI}$ ) for the upper third of the face and calcium hydroxyapatite $(\mathrm{CaHa})$ or hyaluronic acid $(\mathrm{HA})$-based fillers for the medium and lower third; on average we used $50 \mathrm{UI}$ of BoNTA every 6 months, 1.5-3 mL of CaHa filler/year, and $2 \mathrm{~mL}$ of HA filler per year, per patient. Injectables were used in 2-4 sessions.

TCA peeling was performed before BoNTA injections to improve skin quality for first and then treating dynamics lines due to orbiculares could muscle contraction.

HA fillers, with high G', were used when subcutaneous facial volume restoration was required, deep injections with a needle were performed; $\mathrm{CaHa}$ fillers were preferred when tightening secondary to skin stimulation CaHa-mediated was required, cross funning injections in deep dermal layer were performed [5], [6].

Once finished the three steps, the patient was treated monthly with intradermal injections of non-crosslinked $\mathrm{HA}$ and non-ablative radiofrequency sessions.

Table 1: Three-step treatment flow-charts: Based on facial aging appearance, patients were divided in low and moderate/high degree of facial aging, and followed these two different flow-charts

\begin{tabular}{|c|c|c|c|c|c|}
\hline Facial aging appearance & I step & II step & III step & If needed & Maintenance \\
\hline $\begin{array}{l}\text { Low degree of facial } \\
\text { aging (patients presenting } \\
\text { superficial lines and/or skin } \\
\text { spots and/or low degree of } \\
\text { facial soft tissue ptosis, etc.) }\end{array}$ & $\begin{array}{l}\text { Daily retinol } 1 \% \text { cream } \\
\text { application at home } \\
\text { Duration: } 6-8 \text { weeks }\end{array}$ & $\begin{array}{l}10 \% \text { TCA full-face peeling } \\
\text { (performed once a year) } \\
\text { This step is performed, usually, } \\
\text { as soon as daily retinol cream } \\
\text { application has finished }\end{array}$ & $\begin{array}{l}\text { Facial injectables evaluation } \\
\text { Upper third: BoNTA } 50 \mathrm{U} \text { every } 6 \text { months } \\
\text { Mid-low third: } 1.5 \text { to } 3 \mathrm{~mL} \text { of CaHa filler per year, } \\
\text { and a maximum } 2 \mathrm{~mL} \text { of HA filler per year } \\
\text { This step is performed, usually, } 1 \text { month after TCA } \\
\text { peel and } 2-4 \text { injecting sessions were performed }\end{array}$ & Thread lifting & $\begin{array}{l}\text { Monthly intradermal } \\
\text { injections of non-cross- } \\
\text { linked HA and non- } \\
\text { ablative radiofrequency } \\
\text { sessions }\end{array}$ \\
\hline $\begin{array}{l}\text { Medium/High degree of } \\
\text { facial aging (patients } \\
\text { presenting deep facial folds } \\
\text { and/or skin spots and/or } \\
\text { medium-high degree of } \\
\text { facial ptotic tissue, etc.) }\end{array}$ & $\begin{array}{l}\text { Daily retinol } 1 \% \text { cream } \\
\text { application at home } \\
\text { Duration: } 8 \text { to } 10 \\
\text { months }\end{array}$ & $\begin{array}{l}20 \text { to } 30 \% \text { TCA full-face peeling } \\
\text { (performed once a year) } \\
\text { This step is performed, usually, } \\
2 \text { months after starting retinol } \\
\text { cream application }\end{array}$ & $\begin{array}{l}\text { Facial injectables evaluation } \\
\text { Upper third: BoNTA } 50 \mathrm{U} \text { every } 4 \text { months } \\
\text { Mid-low third: } 4.5-7.5 \mathrm{~mL} \text { of CaHa filler per year, } \\
\text { and a maximum of } 3 \mathrm{~mL} \text { of HA filler per year } \\
\text { This step is performed; usually, } 1-2 \text { months after } \\
\text { TCA peel and } 2 \text { to } 4 \text { injecting sessions were } \\
\text { performed }\end{array}$ & & $\begin{array}{l}\text { Monthly intradermal } \\
\text { injections of non-cross- } \\
\text { linked HA and non- } \\
\text { ablative radiofrequency } \\
\text { sessions }\end{array}$ \\
\hline
\end{tabular}




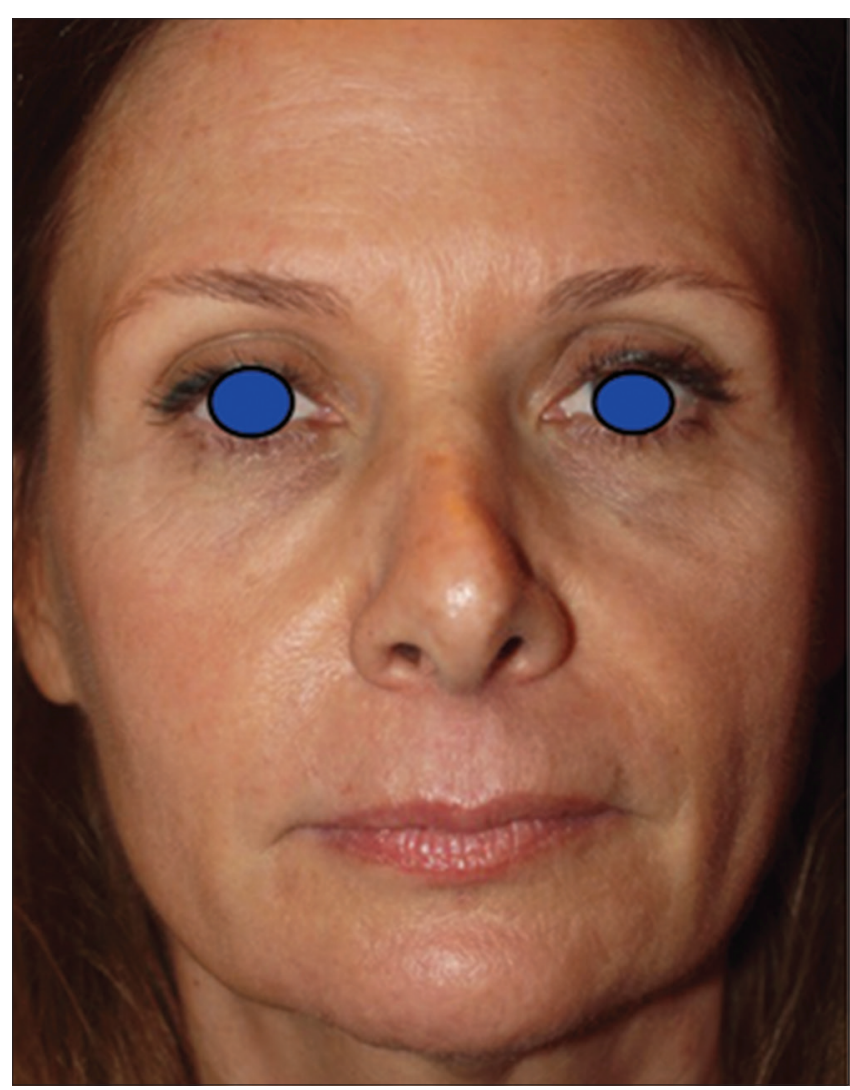

Figure 1: Patients considered with low degree of facial aging were under 50 year's old, with low facial volume loss, superficial lines, and low degree of facial ptosis

When a high degree of facial aging was present, also in these patients, the protocol begins with daily retinol cream application at home, but in this group, the application is prescribed for a longer period (8-10 months); in these cases, the second and the third steps are performed concurrently with the first one. Two to 3 months apart from retinol cream application, a $20 \%$ or $30 \%$ (based on clinician's evaluation) TCA-based peeling (controlled depth Peel by ZO Medical; Health Inc., Irvine, CA) is performed while the patient is still applying at-home retinol cream. After 30-60 days from the peeling, as for the other group of patients, for the third step, while the patient is still applying retinol cream at home (the whole period of application in this group is $8-10$ months), we evaluate the need for injectables; BoNTA (incobotulinum toxin $50 \mathrm{UI}$ ), used for the upper third, is usually injected every 4 months, for medium and lower third soft tissue restoring, CaHa- or HA-based filler are used. On average, we use $4.5-7.5 \mathrm{~mL}$ of $\mathrm{CaHa}$ based filler per year, but the overall volume injected in a year is usually divided into 2-3 injecting sessions based on clinician's and patient's desires, and a maximum of $3 \mathrm{~mL}$ of HA-based filler per year is also used, also in this case different sessions were performed when needed; once soft tissue volume has been restored, if there is the persistence of ptotic tissue we performed a polylactic acid and polycaprolactone ( $\mathrm{pLA}-\mathrm{CL}$ ) anchored thread lifting using, on average, three threads per side. Moreover, in this group of patients, once gained the

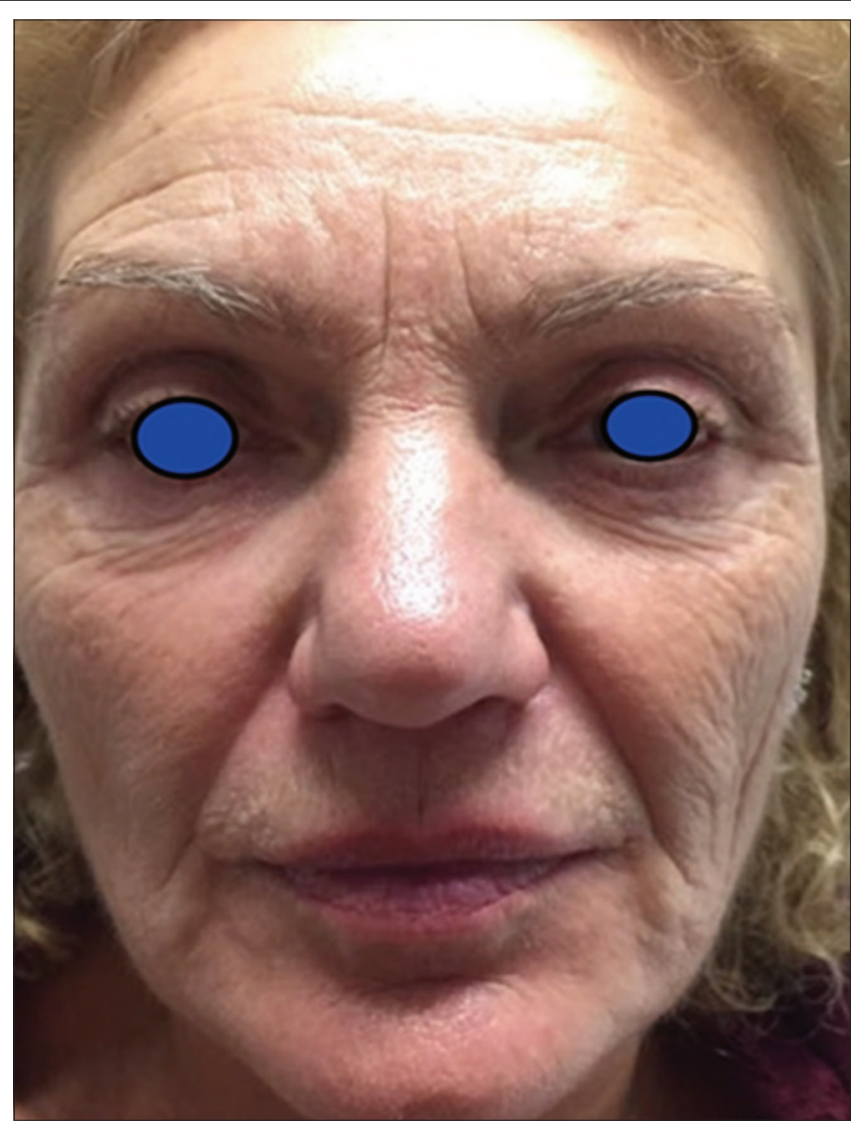

Figure 2: Patients considered with medium/high degree of facial aging were over 50 year's old presenting, with medium/high facial volume loss, deep folds, and medium/high degree of facial ptosis

desired result, the patient was treated monthly with intradermal injections of not cross-linked HA and nonablative radiofrequency sessions.

Results were collected with the A-One Smart system and were represented as summary tables of the metrics assessed at the first visit (TO) and the last visit (T1). For each patient, pre- and post-treatment findings were compared in terms of the percentage change in each variable.

\section{Results}

\section{Demographic data}

The mean age of the 44 patients (42F, $2 \mathrm{M})$ at study entry was 41.7 years; $23(21 \mathrm{~F}, 1 \mathrm{M})$ presenting a low degree and 21 (21F, $1 \mathrm{M}$ ) with medium/high degree of facial aging. The mean treatment duration (i.e., from first [T0] to last [T1] visit) was 159.9 days, 3 months on average for the first group and 8 months on average for the second one (Figure 3a-c, 4a-c). All patients were evaluated with A-One Smart automated skin analysis system (Bomtech Electronics, Seoul, South Korea) during each follow-up visits to detect a change in specific variables such as skin elasticity and hydration, 


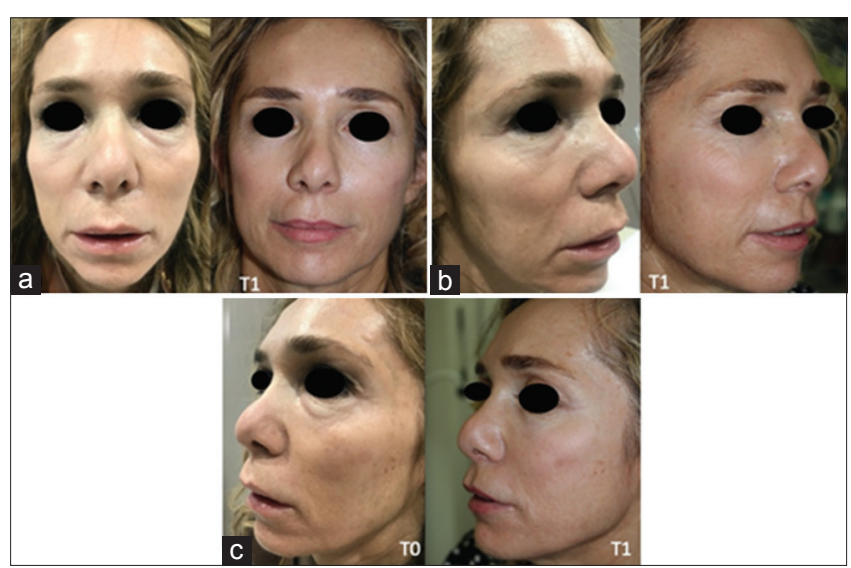

Figure 3: (a-c) (a) Frontal, (b) three-quarter right, and (c) three quarter left views of a 46-year-old lady with a low degree of facial aging. T0 on the left and $T 1$ on the right side of the pictures. The patient applied for 8 weeks retinol cream at home; after "at home treatment," as the second step performed a 10\% TCA peel. One month later, $50 \mathrm{U}$ of BoNTA were used for the upper third (repeated after 6 months), and $1.5 \mathrm{~mL}$ of CaHA and $1 \mathrm{~mL}$ of HA were used to enhance middle and lower third of the face. At the 4th month, patients received a monthly intradermic injection of non-cross-linked HA and non-ablative radiofrequency sessions. T1 picture has been taken 12 months apart from the beginning of at home application of retinol cream

facial pores, and superficial wrinkles; a comparison between T0 and T1 was performed for all the patients.

\section{Clinical Features}

In all the patients of both groups, skin elasticity and hydration improved; at the same time, skin hyperpigmentation, superficial facial wrinkles, and facial pores were reduced; Table 2 depicts the changes in facial parameters in patients who underwent 3D stepwise facial rejuvenation.

Table 2: Changes in facial parameters in patients who underwent 3D stepwise facial rejuvenation.

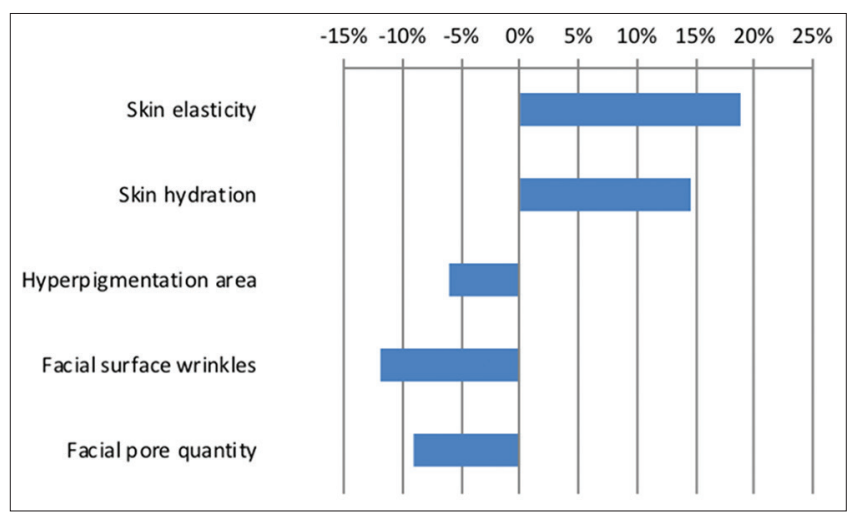

Significant improvements were noted in all metrics assessed. Specifically, skin elasticity (mean \pm standard deviation [SD]) increased by $18.90 \% \pm 2.39 \%$; skin hydration increased by $14.6 \% \pm 0.1356 \%$; the area of facial hyper-pigmentation decreased by $6 \% \pm 0.1613 \%$; surface wrinkles decreased by $12 \% \pm 0.114 \%$, and the quantity of facial pores was lowered by $9 \% \pm 0.0858 \%$.

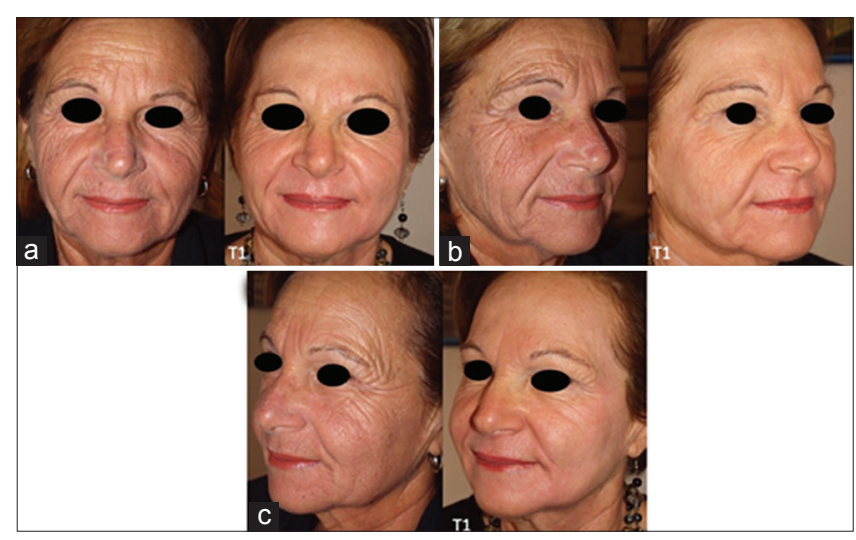

Figure 4: (a-c) (a) frontal, (b) three-quarter right, and (c) three-quarter left views of a 64-year-old lady with moderate/high degree of facial aging; TO on the left and T1 on the right side of the pictures. The patient applied for 8 months retinol cream at home; after 4 months from the "at home treatment," a 20\% TCA peel was performed. At the 5th month, $50 \mathrm{U}$ of BoNTA injections were performed (repeated after 4 months), and between the 6th and the 9th month, $3 \mathrm{~mL}$ of $\mathrm{CaHa} e$ $1 \mathrm{~mL}$ of HA fillers were injected to reshape the middle and lower third of the face. Since the 12th months, the patients received a monthly, intradermic injection of non-cross-linked $H A$ and non-ablative radiofrequency sessions. T1 picture has been taken 18 months apart from the beginning of at home application of retinol cream

Measures taken at $\mathrm{T} 0$ and $\mathrm{T} 1$ with $\mathrm{A}$-one smart automated skin analysis system. Skin elasticity increased by $18.90 \% \pm 2.39 \%$; skin hydration increased by $14.6 \% \pm 0.1356 \%$; the area of facial hyperpigmentation decreased by $6 \% \pm 0.1613 \%$; surface wrinkles decreased by $12 \% \pm 0.114 \%$; and the quantity of facial pores was lowered by $9 \% \pm 0.0858 \%$.

\section{Discussion}

With drastic increase in the popularity of nonsurgical techniques, it is very important to approach patients seeking enhancement and/or rejuvenation with a soft modality respectful of their anatomy and age. Physicians must be guided by the safety for the patient and by an esthetic approach, respectful of proportions [7], [8]. Aging of the face is characterized by different phenomena happening at more or less the same time: Variable skin atrophic changes and wrinkle formation caused by genetic, actinic, and environmental factors; bone volume and facial fat loss; and skin sagging. These issues should be addressed with an elastic approach to achieve natural results [7], [8]. In the present paper, we suggest a customized holistic approach, starting from skin treatments [9], followed by outpatient treatments of esthetic medicine. We divide the patients in two groups, based on aging degree: Low degree of facial aging (patients with superficial lines, skin spot, a low degree of facial soft tissue ptosis, etc.), and high degree of facial aging (deep facial folds, skin spot, medium-high degree of facial ptotic tissue, 
etc.). Outcomes were evaluated, for both groups, with an A-One Smart automated skin analysis system. For both groups of patients, the first step was represented by skincare treatments performed daily at home with the application of daily retinol $1 \%$ cream, prescribed for 6-8 weeks in the first group and for 8-10 months in the second group. Retinoic acid has rejuvenating effects on the stratum corneum and deeper layers of the skin; topically applied tretinoin is the preferred treatment for disorders of the stratum corneum and/ or deep epidermis because it has been shown to repair damaged keratinocytes and increase the rate of mitosis in basal cells; these processes improve skin hydration [10], [11], [12]. Retinol, like retinoic acid, is a derivative of vitamin $A$. In the skin, retinol is oxidized to retinaldehyde and then to tretinoin/retinoic acid, which is the biologically active form of vitamin $A$ [10], [11], [12]. As the second step, performed once finished at the home retinoid acid application in the first group and as overlap treatment in the second group, TCA peeling was performed in both groups, but with different percentage: $10 \%$ for the first group and $20-30 \%$ for the second group. Chemical peels rejuvenate the skin by removing keratin and inactive cells on the skin surface and by helping to stimulate the production of healthy skin cells [13], [14], [15]. In recent years, mediumdepth peeling devices and preparations have been developed; these approaches have minimal risk of side effects if performed as indicated on appropriately diagnosed patients [13]. The third step for each group was represented by a customized approach, both characterized by BoNTA injections, every 6 months for the first group and every 4 months for the $2^{\text {nd }}$ one, followed by facial tissue enhancement with fillers performed at least in 2 sessions during the year. In the second group, thread lifting can be considered if sagging skin is still present after filling procedures. For both groups, once followed the "flow chart," intradermal injections of non-cross-linked $\mathrm{HA}$ and non-ablative radiofrequency sessions were performed monthly during the time. Patients who underwent 3D stepwise facial rejuvenation had improvements in all examined domains of facial aging, including skin elasticity and hydration; moreover, reduction of superficial wrinkles and numbers of visible pores was detected, as for the extent of hyperpigmentation. Harmonious, naturalappearing results were achieved with this facial rejuvenation technique. Patient photographs obtained before and after treatment depicted improvements in skin quality, brightness, texture, and skin spots. At the initial consultation, the practitioner should perform a careful clinical examination to ensure that the treatment plan is suitable to the patient's needs and esthetic expectations [16], [17], [18]. In this study, the A-One Smart system was applied to enable quantitative evaluation of patient photographs. Findings of this research suggest that, in healthy patients, a holistic approach carried out with cosmeceutical treatments applied at home (e.g., retinoic acid and retinol) helps to amplify the effects of outpatient esthetic procedures such as those involving energy-based devices (e.g., radiofrequency and lasers) or injection of dermal fillers or BoNTA, performed after skincare treatments. In author's experience, results of the combination treatment of at-home plus outpatient procedures appear to be more favorable than the result of chemical peels, dermal fillers, lasers, or radiofrequency treatments alone; moreover, asking the patient to have at-home treatment associated with outpatient procedures make the patients feel to be involved in the anti-aging process. In a future study, the author plans to compare results for patients who receive 3D stepwise facial rejuvenation with those for patients who undergo outpatient facial treatments but do not apply cosmeceutical products.

\section{Conclusion}

Outcomes of the present article suggest how important is to customize facial anti-aging treatments. Nonsurgical treatments carried out progressively, involving the patient to perform at-home treatments in associations with outpatient procedures, let to achieve facial improvements in terms of increased skin elasticity and hydration, reduction of hyperpigmentation, wrinkles, and pores.

Although the promising results of this research, further blinded randomized studies should be performed to confirm these outcomes.

\section{References}

1. Shaw RB Jr., Katzel EB, Koltz PF, Yaremchuk MJ, Girotto JA, Kahn DM, et al. Aging of the facial skeleton: Aesthetic implications and rejuvenation strategies. PlastReconstr Surg. 2011;127(1):37483. https://doi.org/10.1097/prs.0b013e3181f95b2d PMid:20871486

2. Rohrich RJ, Pessa JE. The fat compartments of the face: Anatomy and clinical implications for cosmetic surgery. Plast Reconstr Surg. 2007;119(7):2219-27. https://doi.org/10.1097/01.prs.0000265403.66886.54 PMid:17519724

3. McGrath JA, Eady RA, Pope FM. Anatomy and organization of human skin. In: Burns T, Breathnach S, Cox N, Griffiths C, editors. Rook's Textbook of Dermatology. $8^{\text {th }}$ ed. Oxford: Wiley-Blackwell; 2010. p. 1-53. https://doi.org/10.1002/9781444317633.ch3

4. Available from: https://www.surgery.org/sites/default/files/ASAPSStats2018_0.pdf. [Last accesses on 2020 May 22].

5. Zerbinati N, Rauso R, Protasoni M, D'Este E, Esposito C, Lotti T, et al. Pegylated hyaluronic acid filler enriched with calcium hydroxyapatite treatment of human skin: Collagen renewal demonstrated through morphometric computerized analysis. Biol Regul Homeost Agents. 2019;33(6):1967-71.

PMid:31920061 
6. Courdeot-Masuyer C, Robin S, Tauzin H, Humbert P. Evaluation of lifting and antiwrinkle effects of calcium hydroxylapatite filler. In vitro quantification of contractile forces of human wrinkle and normal aged fibroblasts treated with calcium hydroxylapatite. J Cosmet Dermatol. 2016;15(3):260-8. https://doi.org/10.1111/ jocd.12215

PMid:26990784

7. Salti G, Rauso R. Facial rejuvenation with fillers: The dual plane technique. J Cutan Aesthet Surg. 2015;8(3):127-33. https://doi. org/10.4103/0974-2077.167264

PMid:26644734

8. Beylot C. Skin ageing-general features of facial ageing and therapeutic choices. Ann Dermatol Venereol. 2019;146(1):41-74. PMid:30581032

9. Pinsky MA. Efficacy and safety of an anti-aging technology for the treatment of facial wrinkles and skin moisturization. J Clin Aesthet Dermatol. 2017;10(12):27-35.

PMid:29399264

10. Bhawan J. Short- and long-term histologic effects of topical tretinoin on photodamaged skin. Int J Dermatol. 1998;37:28692. https://doi.org/10.1046/j.1365-4362.1998.00433. PMid:9585903

11. Kafi R, Kwak HS, Schumacher WE, Cho S, Hanft VN Hamilton TA, et al. Improvement of naturally aged skin with Vitamin A (retinol). Arch Dermatol. 2007;143:606-12. https://doi. org/10.1001/archderm.143.5.606 PMid: 17515510

12. Fluhr JW, Vienne MP, Lauze C, Dupuy P, Gehring W, Gloor M. Tolerance profile of retinol, retinaldehyde and retinoic acid under maximized and long-term clinical conditions. Dermatology.
1999;199 Suppl 1:57-60. https://doi.org/10.1159/000051381 PMid: 10473963

13. Rendon MI, Berson DS, Cohen JL, Roberts WE, Starker I, Wang B. Evidence and considerations in the application of chemical peels in skin disorders and aesthetic resurfacing. $J$ Clin Aesthet Dermatol. 2010;3(7):32-43. PMid:20725555

14. Kessler E, Flanagan K, Chia C, Rogers C, Glaser DA Comparison of alpha- and beta-hydroxy acid chemical peels in the treatment of mild to moderately severe facial acne vulgaris. Dermatol Surg. 2008;34(1):45-50. https://doi. org/10.1111/j.1524-4725.2007.34007.x PMid: 18053051

15. Camacho FM. Medium-depth and deep chemical peels. J Cosmet Dermatol. 2005;4(2):117-28. PMid: 17166210

16. Harrar H, Myers S, Ghanem AM. Art or science? An evidencebased approach to human facial beauty a quantitative analysis towards an informed clinical aesthetic practice. Aesthetic Plast Surg. 2018;42(1):137-46. https://doi.org/10.1007/ s00266-017-1032-7

PMid:29313062

17. Barca I, Novembre D, Cordaro R, Lo Faro C, Colangeli W, Boschetti CE, et al. Myoepithelioma of the parotid gland: A case report with review of the literature. Oral Maxillofac Surg Cases. 2020;6(1):100131. https://doi.org/10.1016/j.omsc.2019.100131

18. Esposito L, Razzano S, Lo Faro C, Orabona GD, Schonauer F. Pinna fillet flap after advanced external ear tumor resection. JPRAS Open. 2016;8:9-13. https://doi.org/10.1016/j. jpra.2016.02.002 